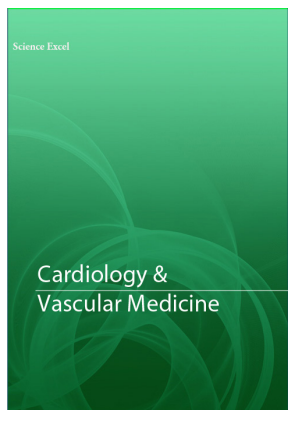

Correspondence

\section{Dr. Katarzyna Kosmalska.}

Department of Cardiology, St.Vincent Hospital, Gdynia, Poland.

E-mail: katarzyn5@wp.pl

- Received Date: 24 April 2020;

- Accepted Date: 04 May 2020;

- Publication Date: 15 May 2020.

Keywords

Prosthetic thrombosis, Embolic stroke, Left atrial appendage closure, Thrombus.

Copyright

(c) 2020 Science Excel. This is an openaccess article distributed under the terms of the Creative Commons Attribution 4.0 International license.

\title{
Complex Cause of Ischaemic Stroke in Patient after Mitral Bioprosthesis Implantation - A Case Report
}

\author{
Katarzyna Kosmalska ${ }^{1 *}$, Andrzej Hrynkiewicz ${ }^{2}$, Krzysztof Jarmoszewicz ${ }^{3}$, and \\ Marcin Fijałkowski ${ }^{4}$ \\ 'Department of Cardiology, St.Vincent Hospital,Gdynia, Poland \\ ${ }^{2}$ Department of Neurology, St.Vincent Hospital, Gdynia, Poland \\ ${ }^{3}$ Department of Cardiac Surgery, Pomeranian Hospital, Wejherowo, Poland \\ ${ }^{4}$ First Cardiology Clinic of Medical University, Gdańsk, Poland
}

\begin{abstract}
We present the history of 60-years-old woman with mitral and aortic bioprosthesis that have been implanted together with left atrial appendage closure. The patient had been treated with VKA and low dose of acetylsali-cylic acid since the surgery. On 28th day of postoperative period an ischemic stroke occurred. In transoesopha-gal echocardiography we have discovered non-obstructive thrombosis of the artificial mitral annulus and a leakiness in left atrial appendage (LAA) closure. There was rapid outflow through the gap in closure directing to mitral annulus in close proximity to visible thrombus. The pathologic flow might have had the contribution in thrombus mobilization and stroke occurrence. We have decided to change anticoagulation to low molecular weight heparin. In control echocardiography, during heparin therapy, thirteen days later, there was remarkable worsening of annulus thrombosis with multiple thrombi present. Re-exchange anticoagulation to warfarin yielded in almost complete resolution of thrombosis after eleven days. We decided to continue anticoagulation over recommended three months not only due to incident of thrombosis but also suspicion of history of atrial fibrillation and presence of disrupted LAA closure.
\end{abstract}

\section{Case Summary}

60 -years-old woman with a history of caseous mitral annulus calcification, severe mitral regurgitation, moder-ate aortic stenosis and regurgitation, have had implantation of mitral and aortic bioprostheses together with left atrial appendage closure. In postoperative period a typical anticoagulation was implemented - low molecular weight heparin followed by vitamin $\mathrm{K}$ antagonist under international normalized ratio (INR) control. Due to coexisting coronary artery disease and venous graft implanted an antiplatelet treatment (75 mg of acetylsali-cylic acid) was also administered. On 28th day after the surgery symptoms of right disparation, ocular ataxia, facial numbness and balance disorders occurred. Ultrasonography and computed tomography angiogram (angi-oCT) revealed an obstruction of right vertebral artery. No visible ischemic focus was discovered in brain com-puted tomography. INR slightly exceeded therapeutic range [1-3]

In transthoracic echocardiography we have discovered turbulent flow through mitral prosthesis with no other dysfunction. Appearance and function of aortic bioprosthesis and other heart structures were normal. In transoesophagal imaging there was small thrombus in lateral part of mitral annulus. Due to incomplete left atrial appendage closure there was a flow through the leak. This outflow was rapid, turbulent and directed straight to mitral annulus, in close proximity to present thrombus. We suspect mobilization of a thrombus re-sulting in stroke by rapid left atrial appendage outflow. After the diagnosis of non-obstructive prosthetic thrombosis, anticoagulation with low molecular heparin was implemented. In control echocardiography, thir-teen days later, we have confirmed not only previously diagnosed thrombus but also four new mobile thrombi. Our next decision was to implement vitamin $\mathrm{K}$ antagonist (VKA) with rigid INR control. After two weeks of the VKA therapy we have observed almost complete resolution of annulus thrombosis. We have continued VKA with target INR 3,0 together with low dose of acetylsalicylic acid for three months. After the three months the anticoagulation was switched to rivaroxaban. Control TOE revealed worsening of thrombosis after one week of rivaroxaban treatment, so we decided to continue further anticoagulation with VKA permanently. Subsequent TOE confirmed the accuracy of VKA treatment, there was only very small, residual thrombus visible. 


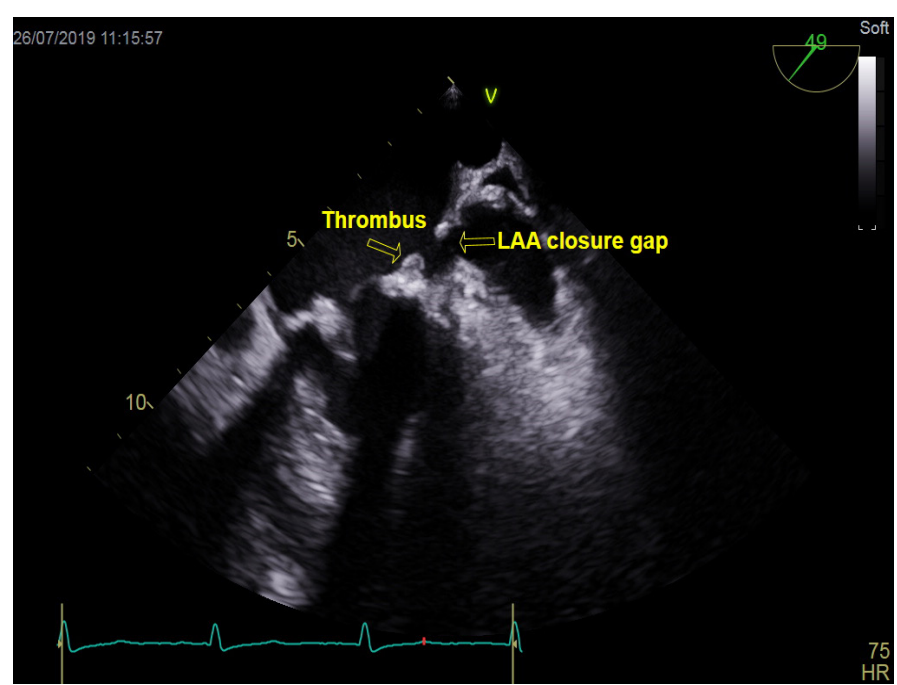

Figure 1: Thrombus on mitral annulus and gap in LAA patch.

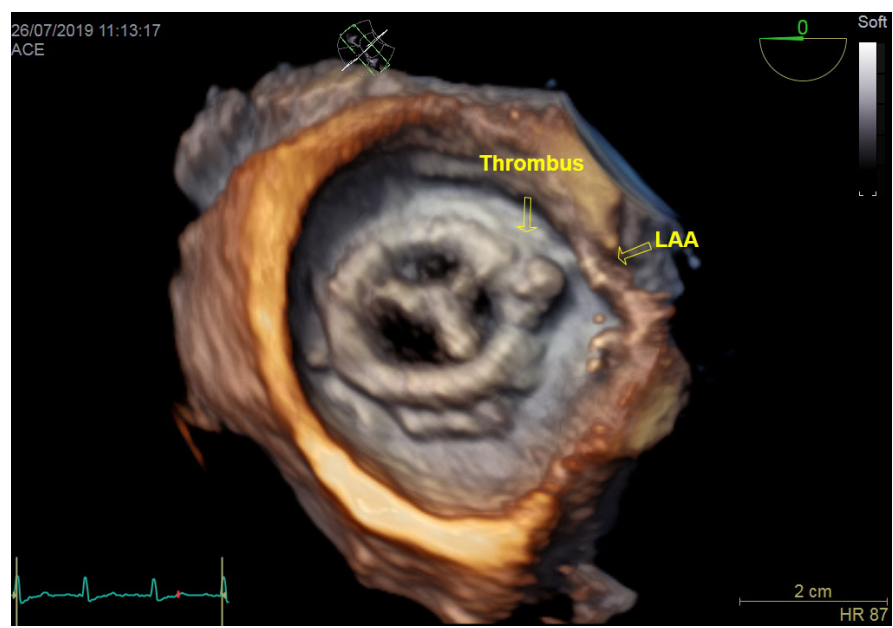

Figure 2: Thrombus on mitral annulus close to LAA in 3D.

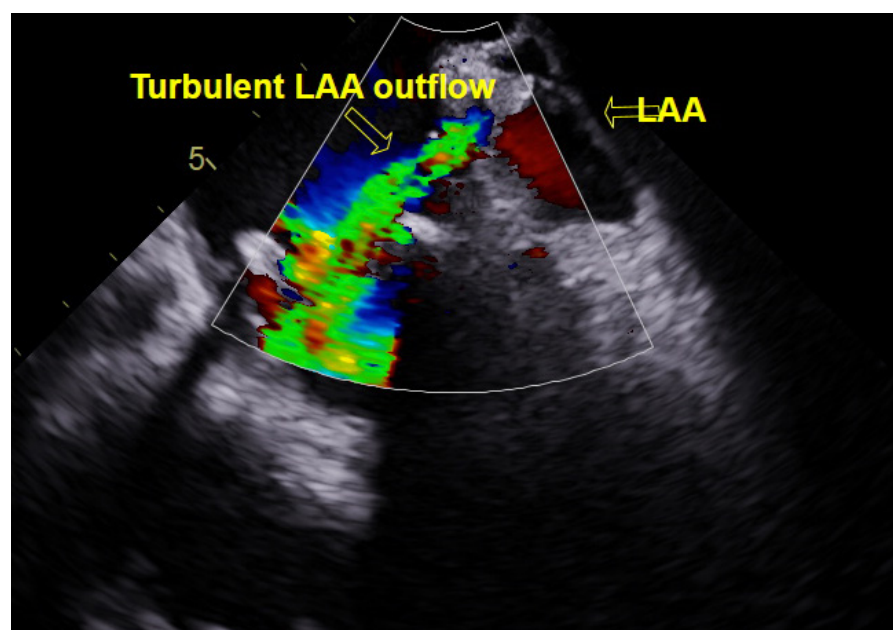

Figure 3: Turbulent LAA outflow directing to mitral annulus.

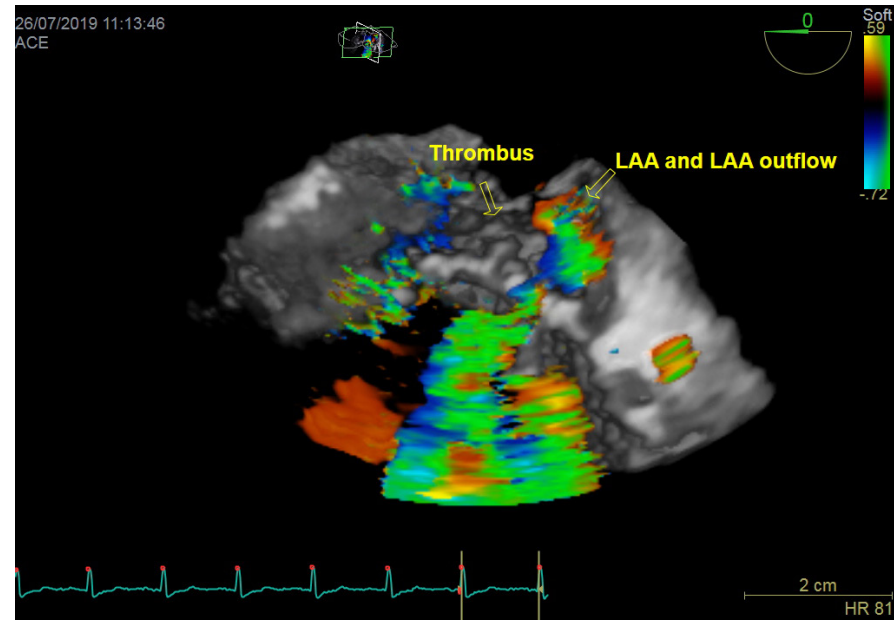

Figure 4: 3D reconstruction pf lateral part of mitral annulus.

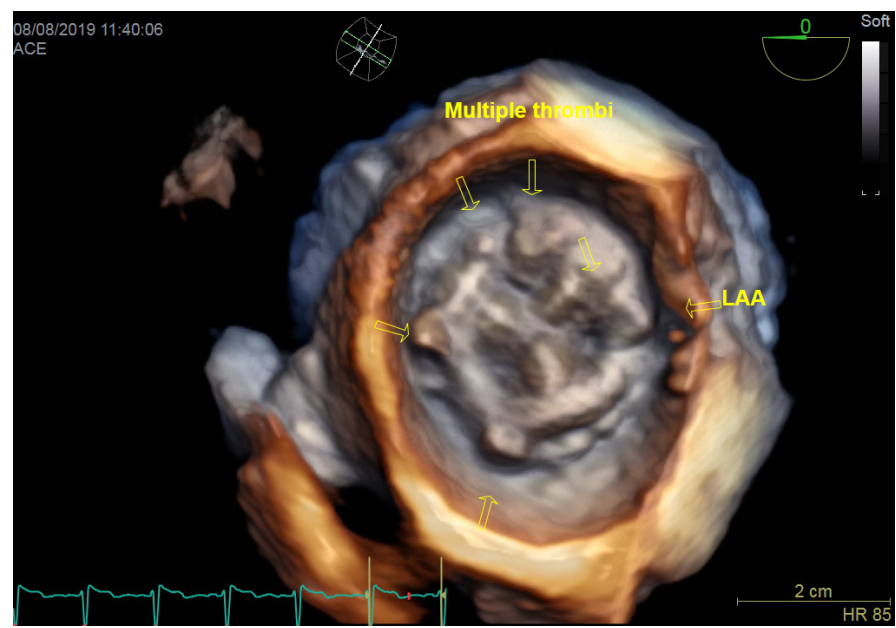

Figure 5: Worsening of annulus thrombosis on low molecular weight heparin therapy.

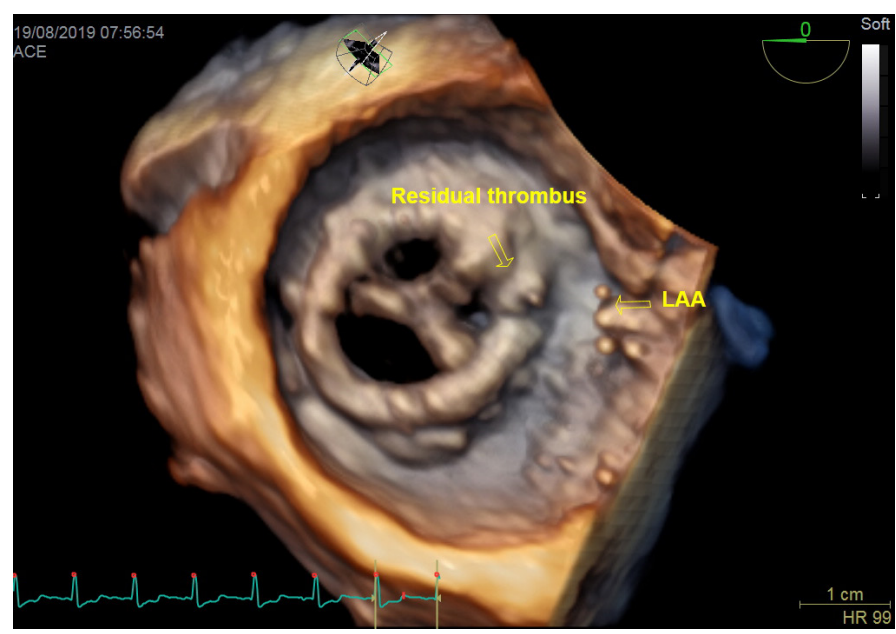

Figure 6: Resolution of thrombosis on vitamin K antagonist therapy. 


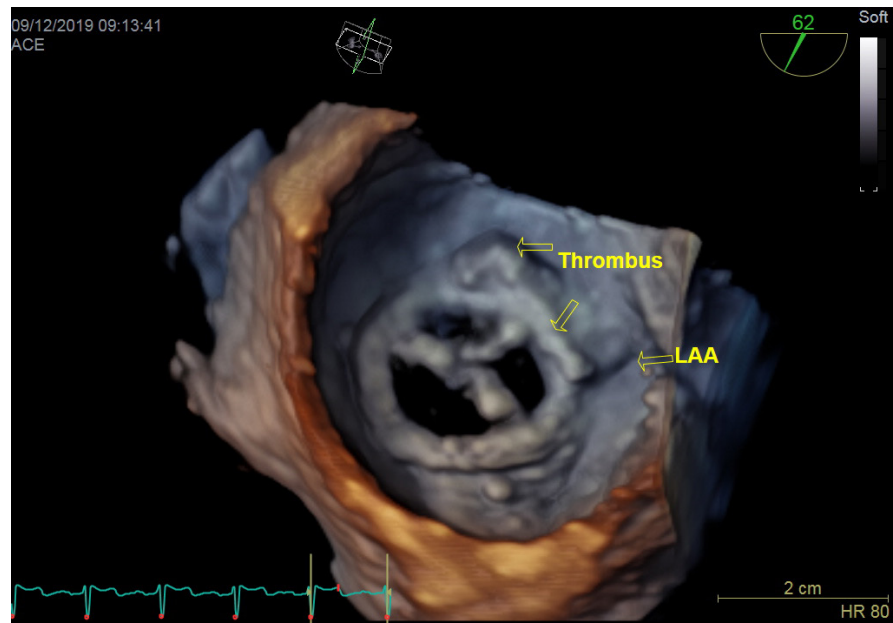

Figure 7: Re-worsening on rivaroxaban 3 months later.

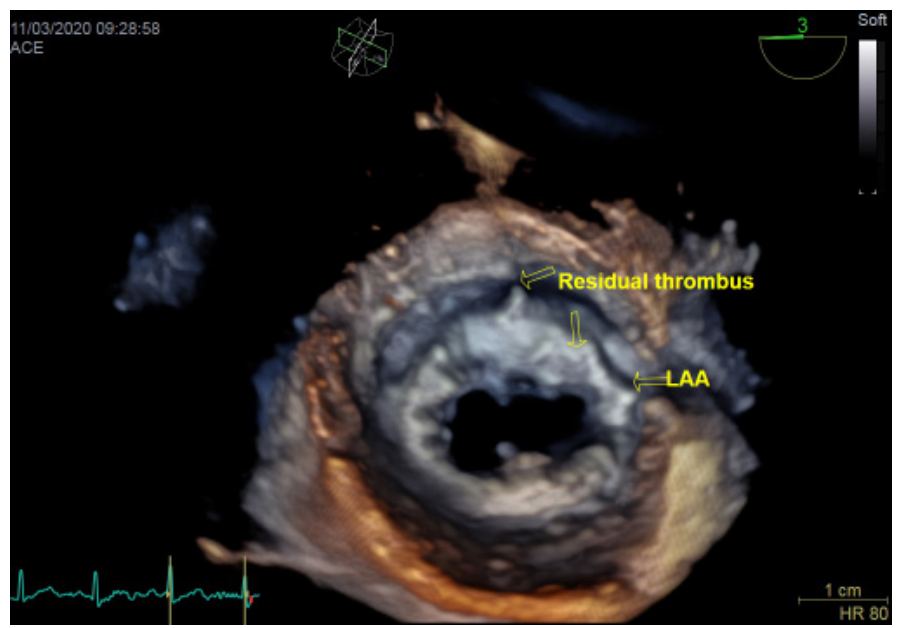

Figure 8: Improvement after returning to vitamin $\mathrm{K}$ antagonist.

\section{Discussion}

Patients after cardiac surgery are high risk group of ischemic stroke [4]. The risk is even higher after valve surgery [5]. Mitral valve replacement presents a $1,62 \%$ threat of stroke in postoperative period [1]. Current recommendations direct us to use vitamin $\mathrm{K}$ anticoagulants for three months after mitral valve replacement (bioprosthesis) $[4,5]$.

Taking into account prevalence of atrial fibrillation in patients subjected to mitral valve surgery and specific surgical access, closure of left atrial appendage emerged as almost a routine [6].

Dispatching of the LAA closure is rare [7]. In our patient it took place probably due to high pressure generated by contracting LAA muscle. Unfortunately outflow through the crack was directing to mitral annulus and prob-ably have mobilized thrombus previously present in this place.
The bare metal of mitral annulus is highly thrombogenous. After the period of epithelialization, (approximate-ly 3 months), thromboembolic risk grows low and the anticoagulation may be stopped.

In this case we think, that continuing of anticoagulation permanently is a good choice. Arguments for further anticoagulation are: thrombembolic event in postoperative period, unclosed left atrial appendage, presence of malign LAA flow that probably interfere epithelialization of mitral annulus, calcification of native mitral an-nulus [2], and suspicion of atrial fibrillation. In this specific case there was no documented atrial fibrillation but the risk of the arrhythmia in patient with the history of severe mitral regurgitation is very high [3]. Vita-min$\mathrm{K}$-antagonists turned out to be better than other anticoagulants: temporary switching to both parenteral antiXa-antagonist (enoxaparin) and oral antiXa-antagonist (rivaroxaban) resulted in rapid worsening of throm-bosis. In both cases, returning to VKA improved the state significantly.

\section{References}

1. Udesh R, Natarajan P, Jeevanantham V, et al. Perioperative Strokes Following Surgical Correction of Mitral Valves: A Systematic Review and Meta-Analysis. Eur Neurol 2017;78:6370 .

2. De Marco M, Gerdts E, Casalnuovo G, et al. Mitral Annular Calcification and Incident Ischemic Stroke in Treated Hypertensive Patients: The LIFE study American Journal of Hypertension 2013; 26(4):567-573.

3. Grigioni F, Avierinos J-F, Ling LH, et al. Atrial fibrillation complicating the course of degenerative mitral regurgitation. J Am Coll Cardiol 2002; 40(1):84-92.

4. Hogue CW Jr, Murphy SF, Schechtman KB, et al. Risk Factors for Early or Delayed Stroke After Cardiac Surgery. Circulation 1999;100:642-647

5. Ruel M, Masters RG, RubensDF, et al. Late incidence and determinants of stroke after aortic and mi-tral valve replacement. Ann Thorac Surg. 78(1):77-83.

6. Baumgartner H, Falk V, Bax JJ, et al. 2017 ESC/EACTS Guidelines for the management of valvular heart disease. Eur Heart J 2017; 38(36):2739-2791.

7. Eikelboom JW, Connolly SJ, Brueckmann M, et al. Dabigatran versus Warfarin in Patients with Me-chanical Heart Valves. N Engl J Med 2013; 369:1206-1214

8. Squiers JJ, Edgerton JR. Surgical Closure of the Left Atrial Appendage: The Past, The Present, The Future. J Atr Fibrillation 2018; 10(5): 1642.

9. Lynch M, Shanewise JS, ChangG, et al. Recanalization of the Left Atrial Appendage Demonstrated by Transesophageal Echocardiography. Ann Thorac Surg 1997; 63(6):1774-1775.

10. Bennaghmouch N, de Veer AJWM, Bode K, et al. Efficacy and Safety of the Use of Non-Vitamin K Antagonist Oral Anticoagulants in Patients with Nonvalvular Atrial Fibrillation and Concomitant As-pirin Therapy A Meta-Analysis of Randomized Trials. Circulation 2017; 137(11):1117-1129. 